
Not for reproduction, distribution or commercial use.

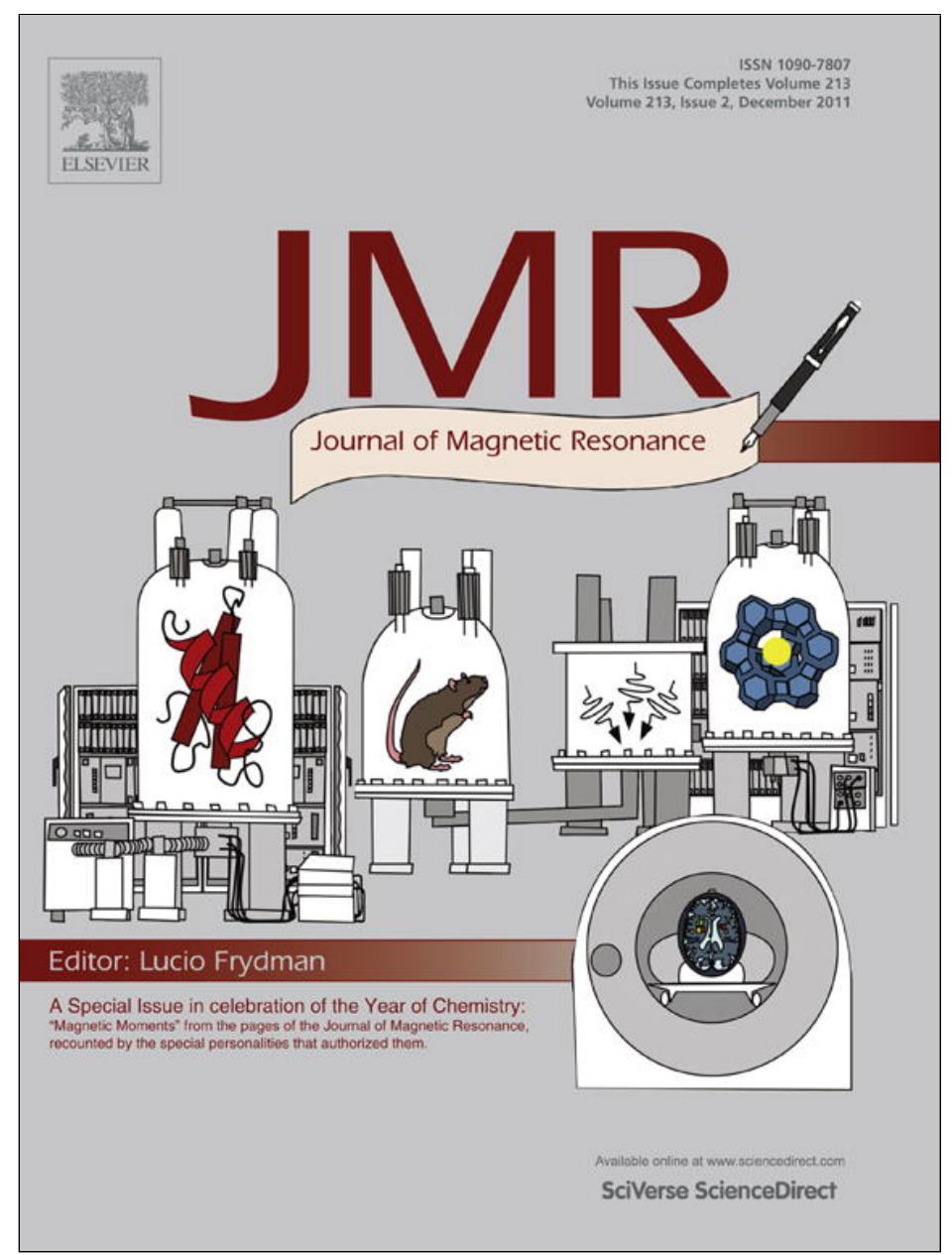

This article appeared in a journal published by Elsevier. The attached copy is furnished to the author for internal non-commercial research and education use, including for instruction at the authors institution and sharing with colleagues.

Other uses, including reproduction and distribution, or selling or licensing copies, or posting to personal, institutional or third party websites are prohibited.

In most cases authors are permitted to post their version of the article (e.g. in Word or Tex form) to their personal website or institutional repository. Authors requiring further information regarding Elsevier's archiving and manuscript policies are encouraged to visit:

http://www.elsevier.com/copyright 
Historical Perspective

\title{
Reflections of pathways: A short perspective on 'Selection of coherence transfer pathways in NMR pulse experiments'
}

\author{
Geoffrey Bodenhausen* \\ Département de Chimie, Ecole Normale Supérieure, 24 Rue Lhomond, 75231, Paris Cedex 05, France \\ Université Pierre-et-Marie Curie, 75005 Paris, France \\ CNRS, UMR 7203, 75005 Paris, France \\ Institut des Sciences et Ingénierie Chimiques, Ecole Polytechnique Fédérale de Lausanne, 1015 Lausanne, Switzerland
}

Interview with the author(s).

A video interview with the author(s) associated with this Historical Perspective and the original article can be found in the online version, at doi:10.1016/j.jmr.2011.08.004.

\section{Introduction}

Initially, Lucio Frydman's invitation to write a few pages about the origins of the 'Selection of coherence transfer pathways' [1] gave me much pleasure, for I tend to regard this paper as my preferred brainchild. It is probably the most influential of the $333 \mathrm{pa}-$ pers that I have been associated with in the last 33 years, and I have greatly enjoyed lecturing about the subject on many occasions, in meetings and classrooms. On second thoughts however, such an enterprise appears fraught with danger. Is it really my brainchild? Of course it isn't! Is it really influential? One should not lay any claims on what others have done with it. Was the lecturing any good? I doubt that my students share my fond recollections.

In the wonderfully open-minded atmosphere of Ray Freeman's Oxford laboratory where I carried out research towards my D. Phil. (1975-1977), we discovered, purely by accident, some artifacts in two-dimensional spin-echo spectra that we affectionately called 'phantoms' and 'ghosts' [2,3]. The former arise when the initial pulse is short of $90^{\circ}$ so that it leaves some longitudinal magnetization, and the refocusing pulse differs from $180^{\circ}$, so that it can excite some transverse magnetization. Ghosts stem from the failure of the refocusing $180^{\circ}$ pulse, at least in some parts of the sample. In our slightly amateurish way, we stumbled upon a method to exorcise these diabolical artifacts, which we dubbed 'Exorcycle' [4], after a movie entitled 'The Exorcist' that was popular at the time. In retrospect, this seems to be the first published example of a phase-cycle, i.e., a series of pulse sequences that are identical except for the relative phases of the radio-frequency pulses. Ever since, we have scrutinized artifacts of all sorts with near-compulsive attention in the hope that they would lead to some new in-

\footnotetext{
* Address: Institut des Sciences et Ingénierie Chimiques, Ecole Polytechnique Fédérale de Lausanne, 1015 Lausanne, Switzerland.

E-mail address: geoffrey.bodenhausen@epfl.ch
}

sights. Hardly had our communication [4] been submitted to the Journal of Magnetic Resonance (Ray had written it up in less than $48 \mathrm{~h}$ ) that David Hoult, having read Ray's lucid description [3] of our beloved phantoms and ghosts, rushed into our Physical Chemistry lab, coming from his Biochemistry lab across the street: '...of course you must realize how you can eliminate these artifacts...' He had invented exactly the same thing! Here is another lesson: if you fail to publish an idea, somebody else will, sooner or later.

When I joined the laboratory of the regretted Regitze Vold and Robert Vold at UCSD, I learned a few things about multiple-quantum coherences, and expanded the 4-step 'Exorcycle' to a 16-step cycle [5]. In modern parlance, one would say: we selected pathways $\Delta p= \pm 4$ instead of $\Delta p= \pm 2$, using $45^{\circ}$ instead of $90^{\circ}$ phase shifts. (It could probably have been done in eight steps, but 'Hexcycle' - short for 'Hexadecacycle' - sounded more devilish than 'Octacycle'!) This was surprisingly effective at removing undesired coherence transfer processes, even when the nominal $180^{\circ}$ refocusing pulse was severely miscalibrated [5]. In a sense, phase cycles appeared to offer an alternative to Malcolm Levitt's composite pulses.

In my days at MIT (1979-1980), David Ruben built a phaseshifting device. Appropriate hardware is now incorporated into routine spectrometers, and I could have done more reading and less tinkering with soldering irons in Oxford, San Diego, MIT and ETH if phase-shifters had been readily available in those days. By the time I returned to ETH (1980-1985), I had become a steadfast believer in the now well-known graphical representations of pathways. I remember that on the occasion of the 125th birthday of ETH, I gave a P. Chem. seminar showing pathways appropriate for an oriented deuteron, which has five levels $(p=-2,-1,0,+1$, +2 ) so that after three pulses there are $5^{3}=125$ possible pathways. Fig. 1 shows a transparency from this early period. Curiously, nobody seems to have followed up on the idea that many 4-step cycles can be replaced without loss by 3-step cycles.

At some point in 1981, Richard Ernst asked me to help complete the manuscript of 'Principles' [6]. At that time, about half of the 


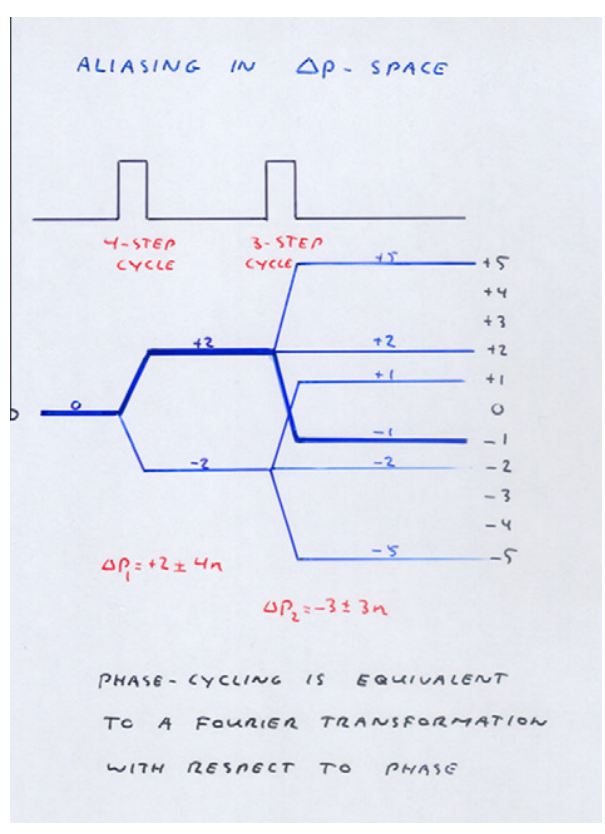

Fig. 1. Transparency prepared for a seminar at ETH in ca. 1982, illustrating how 3step phase cycles might be used to select desired coherence transfer pathways.

book had been written by Richard and Alexander Wokaun. I cannot remember how this came about, but I must have argued that some description of pathways would help to bring the various chapters together. Indeed, chapter 6.3 contains such a description, with extensions to stimulated echoes in chapter 4.6. The formal approach was largely inspired by the classic (though less widely recognized) work of Wokaun and Ernst [7] that defines the order $p=m-m^{\prime}$ of a coherence spanning two eigenstates with magnetic quantum numbers $m$ and $m^{\prime}$, describes the phase behavior of such a coherence in terms of the group of infinitesimal rotations, and rationalizes phase-cycles as Fourier transformations with respect to the phase $\phi$, noting that $p$ and $\phi$ can be considered as conjugate variables, much like time $t$ and frequency $v$, or $\boldsymbol{k}$ vectors and spatial coordinates $\boldsymbol{r}$ in MRI. Among other things, this allows one to understand why several different orders $p, p^{\prime}, \ldots$ are retained simultaneously if the phase increments are too coarse. This results from aliasing or folding in $p$-space, a phenomenon that is familiar to those who have used under-sampling in time-domain.

The task of finishing 'Principles' seemed so hopeless that we decided to submit a separate paper to JMR. A selfless referee wrote three full pages containing no less than 12 elaborate equations of the sort shown in Fig. 2. Wallace Brey, the able Editor of JMR for many years, wrote an elegant letter to Richard Ernst on November 7th 1983 that is still on my files ('I shall, of course, be pleased to accept his manuscript with any revisions you feel it is appropriate to make.').

One may wonder in what respect our 1984 paper [1] represents an improvement over the earlier 1977 work [7]. True, our paper makes allowance for several consecutive coherence transfer steps. It also introduced a rather clumsy notation (that I can never remember myself) using bold and lightface characters with and without brackets to distinguish between selected and rejected pathways. However, the main virtue of the 1984 paper appears

$$
\sigma^{p}\left(t_{i}^{-}\right) \stackrel{U_{i}\left(\varphi_{i}\right)}{\longrightarrow} \sum_{p^{\prime}} \sigma^{p^{\prime}}\left(t_{i}^{+}\right) \exp \left\{-i \Delta p_{i} \varphi_{i}\right\}
$$

Fig. 2. The key equation of our 'pathway' paper. to lie in the graphical representations of coherence transfer pathways, which nicely supplement the information conveyed by pulse sequences. The pathway paper [1] also explains the need to select two mirror-image pathways to obtain pure-phase peaks, as opposed to 'phase-twisted' lineshapes, in two-dimensional spectra [3].

The key equation of our 1984 paper (Fig. 2) says in effect that, if the phase of an external perturbation (i.e., a sequence of pulses) represented by a propagator $U$ is shifted through some arbitrary value $\varphi$, a component of the density operator $\sigma$ having coherence order $p$ before the perturbation is transformed into a superposition of components with coherence orders $p^{\prime}$, the phases of which are determined by the product of the phase $\varphi$ and the difference between the coherence orders $\Delta p=p^{\prime}-p$. For readers who find our original pathway paper [1] a trifle hard to digest, more enjoyable (and more useful) explanations can be found in excellent textbooks by Keeler [8], Levitt [9], and Hore et al. [10].

There are striking similarities between our pathways [1] and Alexander Bain's elegant paper [11]. The latter received about 10 times less credit than ours, judging by the numbers of citations that are so dear to the fans of 'bibliometrics'. This raises a question that may be of some interest to historians of science: why is it that two papers that were published roughly at the same time, and offer similar insight, did not receive equal attention? Somehow, our pathways have become part of widely accepted language, while Bain's work has not quite made it. Mukamel has drawn analogies between our modest pathways and the celebrated Feynman diagrams [12-14]. For such widely used pictorial conventions, the numbers of citations are no more relevant than for Schrödinger's equation.

It is well known that some authors write essentially the same book again and again. Several attempts have been made to generalize pathways to heteronuclear systems, comprising protons $I$, and, say, carbon-13 nuclei $S$. The distinction of coherence orders $p_{I}$ and $p_{S}$ calls for elaborate three-dimensional diagrams. Likewise, Müller designed diagrams for $\mathrm{AX}_{3}$ systems where the three equivalent spins are treated in terms of irreducible tensor operators. Despite their beauty (Fig. 3), such diagrams have not found much popularity [15].

We have tried to generalize pathways to encompass quadrupolar nuclei in anisotropic phases, where one must distinguish between ordinary (Zeeman) echoes and quadrupolar echoes. It turns out to be helpful to make a distinction between coherence orders $p=m^{\prime}-m$ and satellite orders $q=\left(m^{\prime}\right)^{2}-(m)^{2}$. This distinction calls for increasingly baroque coherence transfer pathway diagrams that do not offer sufficient insight to deserve much popularity [16]. We have tried to convince the rapidly growing community of two-dimensional optical 'femtosecond' spectroscopy of the relevance of pathways, through a talk at a symposium organized by Peter Hamm on Rigi Kulm in 2006, and by lecturing to Graham Fleming's group in Berkeley in 2007. To no avail: there appear to be no suitable quantum numbers in optics that bear any useful analogies to our beloved coherence orders $p=m^{\prime}-m$.

It might be wise to heed to Edward Gibbon's eloquent advice: 'I cannot dissemble that six ample quartos must have tried, and may have exhausted the indulgence of the Public; that in the repetition of similar attempts, a successful Author has much more to lose, than he can hope to gain; that I am now descending into the vale of years...' [17].

Clearly, like Gibbon's work, our pathway paper was written with an ambition to set a trend. It contributed to cleaning up a chaotic field of prescriptions for phase cycles that achieved much the same thing under different guises. But it was hardly written to become a citation classic. Had 'bibliometrics' been fashionable in the 1980 s, had granting agencies been as reluctant to subsidize fundamental research as they are today, had they been quicker at shift- 


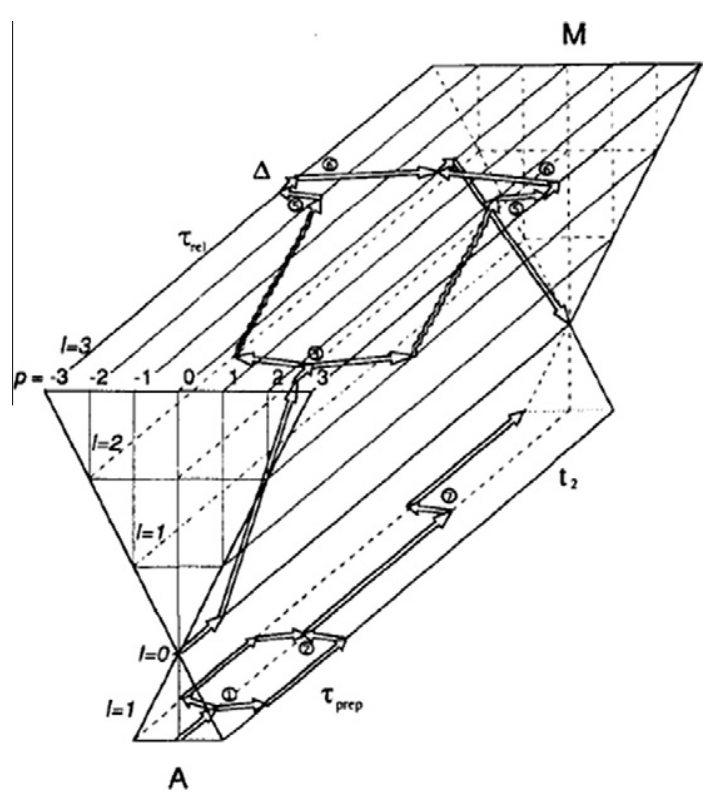

Fig. 3. Coherence transfer diagram appxropriate for an $\mathrm{AX}_{3}$ system in terms, of irreducible tensor operators with ranks $l$ and coherence orders $p$.

ing their focus to 'useful' nanobiotechnology, it appears doubtful that our pathway paper would ever have been written.

\section{Acknowledgments}

The author thanks Richard Ernst, Herbert Kogler, Shaul Mukamel, Norbert Müller and Gareth Morris for their kind advice. Gareth drily characterized the atmosphere in Oxford at the time of 'Exorcycle': "We all of us feel a little defensive at how limited our understanding was in those heady days, but I sometimes wonder whether that didn't aid rather than hinder creativity. It's a mercy that I didn't realise at the time that some of our competitors actually understood what they were doing - I'd probably have taken fright and ended up a synthetic chemist." Let's hope that the community of synthetic chemistry will forgive him these terms of comparison.

\section{References}

[1] G. Bodenhausen, H. Kogler, R.R. Ernst, Selection of coherence transfer pathways in NMR pulse experiments, J. Magn. Reson. 58 (1984) 370-388.

[2] G. Bodenhausen, R. Freeman, G.A. Morris, R. Niedermeyer, D.L. Turner, A simple approach to single-channel quadrature detection, J. Magn. Reson. 25 (1977) $559-562$.

[3] G. Bodenhausen, R. Freeman, R. Niedermeyer, D.L. Turner, Double Fourier transformation in high-resolution NMR, J. Magn. Reson. 26 (1977) 133-164.

[4] G. Bodenhausen, R. Freeman, D.L. Turner, Suppression of artifacts in twodimensional spectroscopy, J. Magn. Reson. 27 (1977) 511-514.

[5] G. Bodenhausen, R.L. Vold, R.R. Vold, Multiple-quantum spin-echo spectroscopy, J. Magn. Reson. 37 (1980) 93-106.

[6] R.R. Ernst, G. Bodenhausen, A. Wokaun, Principles of Nuclear Magnetic Resonance in One and Two Dimensions, Oxford University Press, Oxford, 1987.

[7] A. Wokaun, R.R. Ernst, Selective detection of multiple quantum transitions in NMR by two-dimensional spectroscopy, Chem. Phys. Lett. 52 (1977) 407.

[8] J. Keeler, Understanding NMR Spectroscopy, second ed., John Wiley \& Sons, London, 2010.

[9] M.H. Levitt, Spin Dynamics: Basics of Nuclear Magnetic Resonance, John Wiley \& Sons, London, 2008

[10] P.J. Hore, J.A. Jones, S. Wimperis, NMR: The Toolkit, Oxford Chemistry Primer No. 92, Oxford University Press, 2000.

[11] A.D. Bain, Coherence levels and coherence pathways in NMR - a simple way to design phase cycling procedures, J. Magn. Reson. 56 (1984) 418.

[12] C. Scheurer, S. Mukamel, Magnetic resonance analogies in multidimensional vibrational spectroscopy, Bull. Chem. Soc. Jpn. 75 (2002) 989-999.

[13] C. Scheurer, S. Mukamel, Design strategies for pulse sequences in multidimensional optical spectroscopies, J. Chem. Phys. 115 (2001) 49895004.

[14] C. Scheurer, S. Mukamel, Infrared analogs of heteronuclear nuclear magnetic resonance coherence transfer experiments in peptides, J. Chem. Phys. 116 (2002) 6803-6816.

[15] N. Müller, G. Bodenhausen, Cross correlation of chemical shift anisotropy and dipolar interactions in methyl protons investigated by selective nuclear magnetic resonance spectroscopy, J. Chem. Phys. 98 (1993) 6062-6069.

[16] S. Antonijevic, G. Bodenhausen, Quadrupolar transfer pathways, J. Magn. Reson. 180 (2006) 297-304.

[17] E. Gibbon, in: D. Womersley (Ed.), The history of the decline and fall of the Roman Empire, Penguin, London, 2000, p. 6 (first published 1776). 